\title{
Lesiones Oro-maxilofaciales en el Servicio Médico Legal de Curicó, Chile: Estudio descriptivo
}

\author{
Oro-maxillofacial injuries in the Medical Legal Service of Curicó, Chile: Descriptive study
}

\author{
Loreto Espinosa'; Constanza Faúndez'; Catherine Sandoval'² Saúl Tirado ${ }^{3}$ \& Ignacio Roa ${ }^{4,5}$
}

ESPINOSA, L.; FAUNDEZ, C.; SANDOVAL, C.; TIRADO, S. \& ROA, I. Lesiones oro-maxilofaciales en el Servicio Médico Legal de Curicó, Chile: Estudio descriptivo. Int. J. Odontostomat., 13(4):379-384, 2019.

RESUMEN: Las ocurrencias de lesiones en la región oro-maxilofacial adquieren importancia debido a su complicada anatomía y fisiología, pudiendo resultar en deformidades faciales, adquiriendo interés cuando son causadas por un tercero, pudiendo traer repercusiones legales. El objetivo fue realizar un estudio transversal con el fin de estimar frecuencia y tipificación de lesiones oro-maxilofaciales que requirieron peritaje forense en el Servicio Médico Legal de Curicó, Chile. Se recopilaron datos encriptados de 79 fichas de pacientes entre 17-88 años que realizaron su constatación de lesiones en Servicio Médico Legal de Curicó, Chile, en el lapsus de un año. La frecuencia de lesiones con peritaje forense en la región oro-maxilofacial fue de un $25,82 \%$, provocada principalmente por mecanismo físico. En su mayoría efectuados a individuos del sexo masculino, con un rango etario de entre 20 a 40 años. La violencia interpersonal fue observada como el agente causal más frecuente de lesiones, seguida por accidentes de tránsito. Los sujetos periciados por violencia intrafamiliar, fueron en su totalidad mujeres. Las lesiones más recurrentes fueron fractura y contusión, dentro de ellas encontramos a fractura nasal como la más frecuente, seguida de herida contusa, herida por instrumento cortante, fractura maxilar y por último fractura dental. De la totalidad de las lesiones en estudio solo un tercio estuvieron confinadas exclusivamente en el territorio oro-maxilofacial.

PALABRAS CLAVE: odontología forense, lesión maxillofacial.

\section{INTRODUCCIÓN}

La región oro-maxilofacial resulta ser de gran importancia debido a sus funciones fisiológicas y sensoriales (Bregagnolo et al., 2013), además de contener estructuras vitales tales como sistema nervioso central y vía aérea, así como por el factor estético, pudiendo ser afectado por lesiones que resulten en deformidades faciales (Venegas et al., 2013; Esses et al., 2018). Lesiones que según la literatura son causadas principalmente por accidentes de tránsito, violencia interpersonal y deportes de contacto (DeAngelis et al., 2014).

Este tipo de lesiones se producen habitualmente debido a la exposición y poca protección del segmento corporal (Siber et al., 2015). Las ocurrencias y causas varían dependiendo de la región geográfica.
En nuestro país, los principales factores etiológicos de las lesiones oro-maxilofaciales son la agresión (Venegas et al.) y accidentes de tránsito (González et al., 2015). Desde el punto de vista médico legal, las lesiones adquieren gran interés cuando son causadas por un tercero, pudiendo traer repercusiones legales, tanto en la propia víctima como en el agresor (Fernández \& Arredondo, 2014). En Chile, la institución pública encargada de llevar a cargo las pericias correspondientes para la resolución de los casos es el Servicio Médico Legal (SML) (Caimi \& Caimi, 2013).

Debido a la connotación legal que pueden adquirir algunas lesiones, es que los profesionales de la salud requieren conocimientos legales y elementos éticos que rigen la actividad profesional (Carvallo,

\footnotetext{
${ }^{1}$ Escuela de Odontología, Facultad de Ciencias de la Salud, Universidad de Talca, Talca, Chile.

2 Servicio Médico Legal Región de Aysén del General Carlos Ibáñez del Campo, Chile.

${ }^{3}$ Servicio Médico Legal Curicó, Curicó, Chile.

${ }^{4}$ Departamento de Ciencias Básicas Biomédicas, Facultad de Ciencias de la Salud, Universidad de Talca, Talca, Chile.

${ }^{5}$ Programa de Doctorado en Ciencias Morfológicas, Universidad de La Frontera, Temuco, Chile.
} 
2001), en donde médicos, odontólogos y otras profesionales del área, deben poseer una sólida formación médico legal, con el fin de poder actuar como testigos o peritos. Esto sumado a la judicialización del ejercicio médico y el aumento exponencial de reclamaciones de pacientes por malapraxis a profesionales de la salud, en especial de médicos y odontólogos (González et al., 2005).

La escasa literatura en el tema, no permite observar resultados sobre la frecuencia de lesiones que requirieron peritaje forense en la región oromaxilofacial. La información existente solo da a conocer la epidemiologia según agente traumatizante, rango etario, sexo, entre otros. En base a esto se ha propuesto hacer un estudio transversal de la frecuencia de lesiones oro-maxilofaciales que requirieron peritaje forense en el Servicio Médico Legal de Curicó, Chile, durante el periodo de 1 año, con el objetivo de analizar la frecuencia y tipificación de las lesiones oromaxilofaciales con peritaje forense en el Servicio Médico Legal de Curicó, Chile, desde mayo del 2017 a mayo del 2018.

\section{MATERIAL Y MÉTODO}

El estudio realizado fue de tipo descriptivo observacional transversal, realizado durante un periodo de 1 año, desde mayo del año 2017 a mayo del 2018, cuyo objetivo fue determinar la frecuencia de las lesiones en la región oro-maxilofacial que necesitaron peritaje del Servicio Médico Legal de Curicó, Chile.

Los sujetos de estudio incluyeron a todos los individuos que realizaron su constatación de lesiones en el Servicio Médico Legal de Curicó, Chile, durante el periodo descrito anteriormente, siendo de ambos sexos y sin limitación de edad. El total de la población fue de 306 personas, las cuales debían cumplir con los criterios de inclusión que serán señalados a continuación:

- Pericias de lesionología en territorio oro-maxilofacial, en sujetos vivos, realizadas por el SML de Curicó, Chile.

- Territorio oro-maxilofacial a estudiar estará comprendido desde arco supraciliar hasta zona submandibular.

- Peritaje realizado por el mismo perito.

- Realizadas entre las fechas de mayo del año 2017 a mayo del año 2018.

- Peritaje contenga la información de variables de este estudio.
Por otra parte, los criterios de exclusión fueron: a) Todos los que no cumplan los criterios de inclusión $y$, b) Existencia de datos incompletos. Con lo cual se obtuvo, al término de la recolección de datos, un tamaño muestral de 79 sujetos entre los 17 a 88 años de edad. No fue necesaria la confección de consentimiento informado, puesto que los investigadores no tuvieron acceso a datos que pudiera identificar a los individuos, siendo esta información proporcionada directamente por el director técnico del Servicio Médico Legal, el cual fue el único encargado de proporcionar los datos necesarios para la confección del estudio.

El presente estudio fue aprobado por el Comité Ético Científico de la Facultad de Ciencias de la Salud, Universidad de Talca, Chile (Folio: N²01805).

\section{RESULTADOS}

En el presente estudio el total de personas a las que se les realizó la constatación de lesiones por medio del SML es de 306 individuos. Al aplicar los criterios de inclusión se obtuvieron 89 individuos, y al aplicar criterios de exclusión fueron excluidos 10 a causa de que poseían datos incompletos, por lo cual el total sujetos con los cuales se realizó el estudio fue de 79 , abarcando ambos sexos, entre los 17 a $88(43,1)$ años de edad.

Se presentó una frecuencia de un 25,82 \%, de ocurrencia de lesiones en la región oro-maxilofacial; siendo más frecuentes en hombres $(63,29 \%)$ contra un $36,71 \%$, presente en mujeres, con un rango etario para ambos sexos de entre 21 a 40 años la edad (Tabla I).

Los tipos de lesiones encontradas durante el análisis de datos fue un total de 17 injurias diferentes, las cuales son las siguientes: Fractura nasal/maxilar/ orbitocigomática/orbitaria (techo, piso, pared lateral y pared medial)/mandibular/cigomática/Nasal-OrbitalEtmoidal (NOE) y Le Fort III; Lesiones contusas: Equimosis, escoriación, hematoma, heridas contusas; Traumatismos dentales, tal como subluxación, avulsión y fractura radicular; Heridas por arma de fuego; Heridas por instrumentos cortantes y corto-contundentes; Quemadura de tercer grado. Las lesiones que ocurrieron frecuentemente fueron; herida contusa, heridas por instrumentos cortantes, fractura maxilar y fractura dental. Las lesiones que ocurrieron con menor frecuencia son: Fractura cigomática/NOE/Le Fort 
Tabla I. Frecuencia y tipificación de lesiones oro-maxilofaciales en el Servicio Médico Legal de Curicó, Chile.

\begin{tabular}{lcc}
\hline & Recuento & Porcentaje (\%) \\
\hline Sexo & 50 & \\
Masculino & 29 & 33,29 \\
Femenino & 79 & 100 \\
Mecanismo productor, físico & 66 & 83,54 \\
$\quad$ Objeto contuso & & \\
Agente causal & 42 & 53,16 \\
Violencia interpersonal & $(7)$ & $(8,68)$ \\
(Violencia intrafamiliar) & 30 & 71,43 \\
Golpes (mano-pie) & 34 & 43,04 \\
Accidente de tránsito & & \\
Lesiones & 26 & 29,55 \\
Fractura & 1 & 1,27 \\
Nasal & 1 & 1,27 \\
Cigomática & 1 & 1,27 \\
Naso-orbito-etmoidal & 1 & 1,27 \\
Le Fort III & & \\
Orbitocigomática & 25 & 31,65 \\
Contusión & 21 & 25,58 \\
Herida contusa & 6 & 7,59 \\
Herida por instrume nto cortante & 5 & 6,33 \\
Traumatismo dental & 5 & 6,33 \\
Mixto & 2 & 2,53 \\
Herida por instrumento corto-contundente & 1 & 1,27 \\
Heridas por arma de fuego & 1,27 \\
Quemadura de tercer grado & 62,03 \\
Lesiones concomitantes & &
\end{tabular}

lesiones. Para calcular el porcentaje en la base de datos, se realizó un análisis individual de cada uno, por ejemplo: para el recuento de herida contusa, se escribió solo "herida contusa" borrando las demás lesiones existentes en el mismo sujeto, lo cual fue realizado para saber cuántas veces ocurrió dicha lesión en los individuos peritados, y así sucesivamente con las demás lesiones.

Con respecto al agente causal, el más frecuente fue violencia interpersonal, seguido de accidentes de tránsito (Tabla I), tendiendo este último a ser más frecuente en sexo masculino. Por su parte, en la violencia interpersonal, fue observada como agente causal principal, los golpes (puño, pie, otros), seguido de arma blanca y arma de fuego. También se pudo pesquisar la existencia de violencia intrafamiliar entre las injurias analizadas, con un total de 7 ocurrencias $(8,68 \%)$, presentadas en su totalidad en mujeres.
III/orbitocigomática/pared medial, lateral y posterior de orbita; quemadura de tercer grado; heridas causadas por perdigón y subluxación dental (Tabla I).

Otras lesiones de tejidos blandos como equimosis, escoriación, hematoma, no serán analizadas estadísticamente, puesto que para que exista una lesión de las descritas anteriormente, debe existir de base una lesión en tejido blando, y en muchos de los datos no estaba especificado detalladamente dichas

Tabla II. Ubicación de las lesiones en el territorio maxilofacial.

\begin{tabular}{lcc}
\hline Ubicación maxilofacial & Recuento & Porcentaje (\%) \\
\hline Maxilar & 17 & 19,32 \\
Mandíbula & 6 & 6,82 \\
Nasal & 16 & 18,18 \\
Orbita & 11 & 12,5 \\
Preauricular & 4 & 4,55 \\
Policontuso & 34 & 38,64 \\
Total & 79 & 100 \\
\hline
\end{tabular}

Dentro del territorio maxilofacial se pudieron distinguir diversas ubicaciones anatómicas para las injurias, en donde la policontusión incluye lesiones ubicadas en múltiples sitios en un mismo sujeto, siendo esta la más frecuente en los registros de constatación de lesiones del SML. También existieron lesiones con localización única, donde la más frecuente es la maxilar, seguida de la nasal (Tabla II).

En el presente estudio hubo un único mecanismo causante de las injurias, el cual fue de tipo físico en un $100 \%$ de los sucesos, destacando los objetos contusos $(83,54 \%)$ como el principal productor, seguido de arma blanca, arma de fuego y térmicos (Tabla III).

Tabla III. Distribución de frecuencias según mecanismo productor de la lesión.

\begin{tabular}{llc}
\hline $\begin{array}{l}\text { Mecanismo productor } \\
\text { (Físico) }\end{array}$ & Frecuencia & Porcentaje (\%) \\
\hline Objeto contuso & 66 & 83,54 \\
Arma blanca & 11 & 13,92 \\
Arma de fuego & 1 & 1,27 \\
Acción témica & 1 & 1,27 \\
Total & 79 & 100 \\
\hline
\end{tabular}


ESPINOSA, L.; FAUNDEZ, C.; SANDOVAL, C.; TIRADO, S. \& ROA, I. Lesiones oro-maxilofaciales en el Servicio Médico Legal de Curicó, Chile: Estudio descriptivo. Int. J. Odontostomat., 13(4): 379-384, 2019

\section{DISCUSIÓN}

La lesionología se preocupa del estudio de las lesiones causadas por terceros, con implicancia legal para los individuos involucrados en los incidentes, aplicada tanto a individuos vivos como muertos, ayudando en la identificación de la causal de la lesión, tiempo de evolución de la misma, entre otros (Fernández \& Arredondo). Cuando estas afectan al territorio maxilofacial, adquieren real importancia debido a las estructuras presentes en esta región anatómica y las funciones que éstas realizan (Bregagnolo et al.), así como la posibilidad de generar deformidades faciales (Esses et al.).

En el presente estudio, realizado en el SML de Curicó, Chile, fueron registrados un total de 306 sujetos, quienes realizaron constatación de lesiones durante el periodo de la investigación, de estos solamente el 79 cumplían con los criterios de inclusión, representando un $25,82 \%$ del total. Se dificulta la comparación, de los resultados obtenidos, con otros estudios, debido a la escases de publicaciones en esta área.

Con respecto a la distribución por sexo, la mayoría de los estudios que analizaron lesiones oromaxilofaciales, reportan al género masculino como el más prevalente, tanto en el extranjero (Arabion et al., 2014; Bonavolontà et al., 2017; Morales-Olivera et al., 2017; Teshome et al., 2017), como en Chile (Yoma \& Zúñiga, 2010; Horwood \& Coloma, 2018), siendo coincidentes a los resultados obtenidos en este estudio $(63,29 \%$ sexo masculino versus $36,71 \%$ sexo femenino). Lo cual puede deberse a que el sexo masculino esta principalmente involucrado en los agentes causales de producción de dichas lesiones, como lo son actos violentos, accidentes automovilísticos, entre otros (Horwood \& Coloma).

Al analizar la distribución según edad, de acuerdo a diferentes investigaciones, aquellas con ocurrencia más frecuente de lesiones oro-maxilofaciales fue en individuos menores de 40 años (Venegas et al.; González et al., 2015; Morales-Olivera et al.; Teshome et al.; Esses et al.), lo cual es similar a los resultados obtenidos en el presente estudio, en donde la edad en que estas lesiones son más recurrentes es entre los 20 a 40 años $(43,03 \%)$.

La violencia interpersonal $(53,16 \%)$, fue detectada como el agente causal más frecuente de lesio- nes, seguida por accidentes de transito $(43,04 \%)$, concordante con reportes de investigadores tanto nacionales (Yoma \& Zúñiga; Venegas et al.; Horwood \& Coloma), como internacionales (Fuertes et al., 2010; Morales-Olivera et al., 2017; Teshome et al.). Sin embargo otros estudios, si bien indican a ambos agentes como los más prevalentes, fueron los accidentes de tránsito, los que se presentaron en más oportunidades, seguido de violencia interpersonal (Arabion et al.; González et al., 2015; Sbordone et al., 2018). Por lo cual, con respecto a los agentes causales de las injurias, existe similitud entre los estudios realizados por otros autores, en distintos países, y el nuestro. Las diferencias observadas entre violencia interpersonal y accidentes de tránsito dependen principalmente de las diferencias culturales, socioeconómicas (Morales Navarro \& Vila Morales, 2016) y religiosas (Arabion et al.) del entorno en donde se realice la investigación. Según Kraft et al. (2012), los accidentes de transito son la causa más frecuente de lesiones en países en vías de desarrollo, en cambio la violencia interpersonal se está convirtiendo en la causa más común de injurias en países desarrollados, esto principalmente debido a mejoras de las carreteras, mayor tecnología en los nuevos automóviles e implementación de mayor número de normas de seguridad vial (Morales Navarro \& Vila Morales). La presencia de accidente de transito entre las causas más frecuentes de lesiones, concuerda con los datos de mortalidad a nivel nacional y regional, en donde los accidentes de tránsito son la primera causa de defunción (INE, 2018).

Al referirnos a la violencia intrafamiliar, esta fue clasificada dentro de violencia interpersonal, correspondiente al $16,7 \%(n=7)$, cifra mayor a lo reportado a nivel nacional por Raposo et al. (2013) y Horwood \& Coloma, en los cuales se obtuvo un $1,3 \%$ y $1,79 \%$ del total de los casos, respectivamente, posiblemente la discrepancia entre estos y nuestro estudio, radique en que nosotros utilizamos para nuestros registros cualquier tipo de lesión en el territorio oro-maxilofacial, en cambio Raposo et al. y Horwood \& Coloma, solamente los datos correspondientes a fracturas.

Con respecto al tipo de lesión, las fracturas y lesiones contusas, fueron las observadas con mayor recurrencia, coincidentes a lo observado previamente por Teshome et al. y Yoma \& Zúñiga, respectivamente. Existe discrepancia entre la ubicación más repetitiva de las injurias, según Bonavolontà et al. la localización más frecuente es en mandíbula; por el contrario, otros estudios indican que la ubicación con mayor frecuencia es en maxilar (Bregagnolo et al.; González et al., 2015). 
De las lesiones tipo fractura, descritas en la literatura, la mandibular es la más frecuente (Yoma \& Zúñiga; Raposo et al.; Arabion et al.; Bonavolontà et al.; Horwood \& Coloma) seguido de fracturas del complejo cigomático, (Raposo et al.; Arabion et al.). Sin embargo, de los resultados obtenidos en este estudio, discrepan a lo observado, ya que la lesión más frecuente en nuestros registros fue fractura nasal $(29,55 \%)$, siendo fractura mandibular $(2,53 \%)$ y cigomática $(1,27 \%)$ entre las lesiones con menor frecuencia, la diferencia podría radicar en la recolección del tipo de datos, ya que algunas investigaciones excluían a fractura nasal durante la recolección, lo cual puede ser un factor que provoque tal discrepancia.

Existieron lesiones concomitantes a los traumas maxilofacial en un 19,5 \% según Yoma \& Zúñiga y en el $38 \%$ de los casos según Horwood \& Coloma, estando ubicadas comúnmente en cráneo (traumatismo encéfalo craneano) (Yoma \& Zúñiga; Horwood \& Coloma) y miembros superiores (Yoma \& Zúñiga), encontrándose en menor frecuencia lesiones en abdomen y columna cervical (Yoma \& Zúñiga; Horwood \& Coloma), cifras muy por debajo de lo observado en el presente estudio, donde las lesiones asociadas ocurrieron en un $62,03 \%$, principalmente a nivel del miembro superior, y en menor cantidad en abdomen coincidiendo con Yoma \& Zúñiga.

Un aspecto relevante a señalar, es que a pesar que dentro de los currículos de Odontología, impartidos por las distintas Universidades en Chile, son incorporadas competencias en el área de anatomía del territorio oro-maxilofacial, ética, entre otros, hay carencia en temas relacionados a la odontología legal y forense, haciéndose necesaria su incorporación (Lagos \& Maraboli, 2015), a pesar de que la lesionología es reconocida por la Asociación Chilena de Enseñanza de la Odontología (ACHEO) como un tema fundamental a estudiar por los futuros Cirujanos Dentistas, encontrándose entre los contenidos necesarios y no abordados por la mayoría de las Universidades Estatales Chilenas (Lagos \& Maraboli).

\section{CONCLUSIONES}

Los peritajes requeridos en el SML de Curicó, Chile, fueron en su mayoría efectuados a individuos del sexo masculino, con un rango etario de entre 20 a 40 años. La violencia interpersonal fue observada como el agente causal más frecuente de lesiones, seguida por accidentes de tránsito. Los sujetos periciados por violencia intrafamiliar, fueron en su totalidad mujeres. Las lesiones más recurrentes fueron fractura y contusión, dentro de ellas encontramos a fractura nasal como la más frecuente, seguida de herida contusa, herida por instrumento cortante, fractura maxilar y por último fractura dental. De la totalidad de las lesiones en estudio solo un tercio estuvieron confinadas exclusivamente en el territorio oro-maxilofacial.

ESPINOSA, L.; FAUNDEZ, C.; SANDOVAL, C.; TIRADO, S. \& ROA, I. Oro-maxillofacial injuries in the Medical Legal Service of Curicó, Chile: Descriptive study. Int. J. Odontostomatol., 13(4):379-384, 2019

ABSTRACT: Occurrences of lesions in the oromaxillofacial region acquire importance due to their complicated anatomy and physiology, which may result in facial deformities, acquiring interest when caused by a third party, and may have legal repercussions. The objective was to carry out a crosssectional study in order to estimate the frequency and typing of oro-maxillofacial injuries that required forensic expertise in the Legal Medical Service of Curicó, Chile. Encrypted data was collected from 79 records of patients between 17-88 years who made their findings of injuries in the Medical Legal Service of Curicó, Chile, in the lapse of one year. The frequency of injuries with forensic expertise in the oro-maxillofacial region was $25.82 \%$, caused mainly by physical mechanism. Mostly made to individuals of the male sex, with an age range of between 20 to 40 years. Interpersonal violence was observed as the most frequent causal agent of injuries, followed by traffic accidents. The subjects trained by intrafamily violence were all women. The most recurrent injuries were fracture and contusion, within which we found a nasal fracture as the most frequent, followed by a contusive wound, a cutting instrument wound, a maxillary fracture and finally a dental fracture. Of the totality of the lesions under study, only one third were confined exclusively in the oro-maxillofacial territory.

KEY WORDS: Forensic dentistry, maxillofacial injury.

\section{REFERENCIAS BIBLIOGRÁFICAS}

Arabion, H.; Tabrizi, R.; Aliabadi, E.; Gholami, M. \& Zarei, K. A retrospective analysis of maxillofacial trauma in shiraz, iran: a 6-year- study of 768 patients (2004-2010). J. Dent. (Shiraz), 15(1):15-21, 2014.

Bonavolontà, P.; Dell'aversana Orabona, G.; Abbate, V.; Vaira, L. A.; Lo Faro, C.; Petrocelli, M.; Attanasi, F.; De Riu, G.; laconetta, G. \& Califano, L. The epidemiological analysis of maxillofacial fractures in Italy: The experience of a single tertiary center with 1720 patients. J. Craniomaxillofac. Surg., 45(8):1319-26, 2017. 
Bregagnolo, L. A.; Bregagnolo, J. C.; Silveira, F.; Bérgamo, A. L.; Santi, L. N. \& Watanabe, M. G. Oral and maxillofacial trauma in Brazilian children and adolescents. Braz. Dent. J., 24(4):397-401, 2013.

Caimi, L. \& Caimi, P. Manual de Urgencias Médicoquirúrgicas. Hospital Dr. Gustavo Fricke. $2^{\mathrm{a}}$ ed. Santiago de Chile, Mediterráneo, 2013.

Carvallo, V. A. Consideraciones éticas sobre el error en medicina. Rev. Méd. Chile, 129(12):1463-5, 2001

DeAngelis, A. F.; Barrowman, R. A.; Harrod, R. \& Nastri, A. L. Review article: Maxillofacial emergencies: Maxillofacial trauma. Emerg. Med. Australas., 26(6):530-7, 2014.

Esses, D. F.; Costa, F. W.; Sá, C. D., Silva, P. G.; Bezerra, T. M.; Carvalho, F. S.; de Medeiros, J. R. \& Soares, E. C. Occupational group, educational level, marital status and deleterious habits among individuals with maxillofacial fractures: retrospective study. Med. Oral Patol. Oral Cir. Bucal, 23(1):e13-e22, 2018.

Fernández, A. E. \& Arredondo, F. Z. Guía Practica de Lesiones. Valoración Clínica y sus Implicancias Legales. Barcelona, Elsevier, 2014.

Fuertes, L. F.; Mafla, A. C. \& López, E. A. Análisis epidemiológico de trauma maxilofacial en Nariño, Colombia. CES Odontol., 23(2):3340, 2010.

González, E.; Pedemonte, C.; Vargas, I.; Lazo, D.; Pérez, H.; Canales, M. \& Verdugo-Avello, F. Fracturas faciales en un centro de referencia de traumatismos nivel I. Estudio descriptivo. Rev. Esp. Cir. Oral Maxilofac., 37(2):65-70, 2015.

González, L.; Inzunza, J. A.; Bustos, L.; Vallejos, C. \& Gutiérrez, R. Training and research in forensic medicine: present situation and future challenges for medical schools in Chile. Rev. Med. Chil., 133(7):805-12, 2005.

Horwood, A. F. \& Coloma, O. B. Caracterización de los casos de fracturas maxilofaciales operados en el Hospital Carlos van Buren, Chile, entre los años 2010-2014. Rev. Esp. Cir. Oral Maxilofac., 40(4):169-75, 2018.

Instituto Nacional de Estadísticas (INE). Sitio Web. Santiago de Chile, Instituto Nacional de Estadísticas, 2018. Disponible en: http:// www.ine.cl

Kraft, A.; Abermann, E.; Stigler, R.; Zsifkovits, C.; Pedross, F.; Kloss, F. \& Gassner, R. Craniomaxillofacial trauma: synopsis of 14,654 cases with 35,129 injuries in 15 years. Craniomaxillofac. Trauma Reconstr., 5(1):41-50, 2012.

Lagos, Z. M. \& Maraboli, S. D. Comparación del Nivel de Registro en la Ficha Post Mortem de la Interpol aplicada a Odontólogos Con y Sin Formación en Odontología Forense. Tesis para optar al Título de Cirujano Dentista. Talca, Facultad de Ciencias de la Salud, Escuela de Odontología, Universidad de Talca, 2015.

Morales Navarro, D. \& Vila Morales, D. Aspectos generales del trauma maxilofacial. Rev. Cuba. Estomatol., 53(3):116-27, 2016.

Morales-Olivera, J. M.; Hernández-Ordoñez, R. \& Pacheco-López, R. Estudio epidemiológico del trauma facial en el Servicio de Cirugía Plástica y Reconstructiva del Hospital General «Dr. Rubén Leñero» en la Ciudad de México. Incidencia de 5 años. Cir. Plast., 26(3):11924, 2017.

Raposo, A.; Preisler, G.; Salinas, F.; Muñoz, C. \& Monsalves, M. J. Epidemiología de las fracturas maxilofaciales tratadas quirúrgicamente en Valdivia, Chile: 5 años de revisión. Rev. Esp. Cir. Oral Maxilofac., 35(1):18-22, 2013.

Sbordone, C.; Barca, I.; Petrocelli, M.; Dell'Aversana Orabona, G.; Vaira, L. A.; Colangeli, W.; Cristofaro, M. G.; Giudice, M.; Giudice, A.; Cassandro, F. M.; et al. The influence of socioeconomic factors on the epidemiology of maxillofacial fractures in Southern Italy. $J$. Craniofac. Surg., 29(8):2119-23, 2018.

Siber, S.; Matijevic, M.; Sikora, M.; Leovic, D.; Mumlek, I. \& Macan, D. Assessment of oro-maxillofacial trauma according to gender, age, cause and type of the injury. Acta Stomatol. Croat., 49(4):340-7, 2015.
Teshome, A.; Andualem, G.; Tsegie, R.; \& Seifu, S. Two years retrospective study of maxillofacial trauma at a tertiary center in North West Ethiopia. B. M. C. Res. Notes, 10(1):373, 2017.

Venegas, R. O.; Nicola, S. M.; Barrera, C. R.; Zambra, R. M.; Olivos, B. B. \& Tovar, O. R. Estudio descriptivo del traumatismo máxilofacial en el Hospital de La Serena entre los años 2004 2011. Rev. Chil. Cir., 65(6):525-9, 2013.

Yoma, G. T. \& Zúñiga, S. E. Estudio Epidemiológico del Trauma Cráneofacial en Pacientes Mayores de 15 Años del Servicio de Urgencia del Complejo Asistencial Barros Luco Trudeau, Santiago Chile: Período Enero 2006 a Marzo 2010. Tesis de Pregrado, para optar al Título de Cirujano Dentista. Santiago de Chile, Universidad de Chile, 2010.

Dirección de correspondencia:

Dr. Ignacio Roa Henríquez

Unidad de Morfología

Departamento de Ciencias Básicas Biomédicas

Facultad de Ciencias de la Salud

Universidad de Talca Av. Lircay s/n

Talca

CHILE

Email: iroa@utalca.cl

Recibido : 04-03-2019

Aceptado: 24-06-2019 